\title{
Varieties with quadratic entry locus, II
}

\author{
Paltin Ionescu and Francesco Russo
}

\begin{abstract}
We continue the study, begun in [Rus07], of secant defective manifolds having 'simple entry loci'. We prove that such manifolds are rational and describe them in terms of tangential projections. Using also the work of [IR07], their classification is reduced to the case of Fano manifolds of high index, whose Picard group is generated by the hyperplane section class. Conjecturally, the former should be linear sections of rational homogeneous manifolds. We also provide evidence that the classification of linearly normal dual defective manifolds with Picard group generated by the hyperplane section should follow along the same lines.
\end{abstract}

\section{Introduction}

An $n$-dimensional closed submanifold $X \subset \mathbb{P}^{N}$ is secant defective if its secant variety $S X \subseteq \mathbb{P}^{N}$ has dimension less than $2 n+1$, the expected dimension. The secant defect of $X$ is then $\delta=$ $\delta(X):=2 n+1-\operatorname{dim}(S X)$. Secant defective manifolds naturally fall into two categories. Manifolds admitting 'non-trivial projections' are those for which $S X \neq \mathbb{P}^{N}$, see [Sev01, Zak93], while manifolds 'of small codimension' correspond to the other case, $S X=\mathbb{P}^{N}$, see [BL72]. Let $x, y \in X$ be two general points and let $p$ be a general point on the line $\langle x, y\rangle$. Consider the closure of the locus of secants to $X$ passing through $p$. Its trace on $X$, denoted as $\Sigma_{p}$, is called the entry locus (with respect to $p$ ) and has dimension $\delta$. Our aim is to find classification results for secant defective manifolds whose entry loci are simple enough. Consider embedded manifolds $X \subset \mathbb{P}^{N}$ as above, such that through two general points $x, y \in X$ there passes an $r$-dimensional quadric hypersurface, say $Q^{r}$, contained in $X$. Observe that $Q^{r} \subseteq \Sigma_{p}$ if $p \in\langle x, y\rangle$; in particular $r \leqslant \delta$, see [KS02]. When $r=1$, we call such manifolds conic-connected (CCMs for short). The extremal case $r=\delta$ was called 'manifolds with local quadratic entry locus' (LQELMs), while the special case when $Q^{r}=\Sigma_{p}$ was named 'manifolds with quadratic entry locus' (QELMs); see [KS02, Rus07, IR07]. Being rationally connected, these special classes of secant defective manifolds may be studied in the context of Mori theory; see [Mor79, Mor82, KMM92] and also [Deb01, Kol96, Hwa01]. The interested reader can find further motivation and various examples in the introduction to [Rus07]. The present paper continues the line of investigation started in [Rus07]; see also [IR07]. We acknowledge once more our intellectual debt to the classical work by Scorza; see [Sco08, Sco09]. The content of the paper is described below.

Tangential projections turned out to be a basic tool in the classical works (by Severi, Scorza, Terracini, and others) on secant defective manifolds. In what follows, by the $\delta$-partial tangential projection we mean projection from a $\delta$-codimensional linear subspace (passing through $x$ ) of the projective tangent space at $x \in X$. In Theorem 2.1 we show that the generic $\delta$-partial tangential

Received 23 March 2007, accepted in final form 11 January 2008.

2000 Mathematics Subject Classification 14N05, 14M99, $14 \mathrm{~J} 45$.

Keywords: secant defective, quadratic entry locus manifold, tangential projection, conic-connected, dual defective.

The first author is partially supported by the Italian Programme 'Incentivazione alla mobilità di studiosi stranieri e italiani residenti all'estero'. The second author is partially supported by CNPq (Centro Nacional de Pesquisa), Grants 300961/2003-0, 308745/2006-0 and 474475/2006-9, and by PRONEX/FAPERJ-Algebra Comutativa e Geometria Algebrica.

This journal is (C) Foundation Compositio Mathematica 2008. 


\section{P. IONESCU AND F. Russo}

projection of a QELM is birational. The proof is based on a degeneration technique introduced in [CMR04] (see also [CR06]). The key point is that the general fibre of the (full) tangential projection is a degeneration of the general entry locus. Conversely, if the generic $\delta$-partial tangential projection of $X$ is birational, then $X$ is a LQELM. Moreover, we show that defective LQELMs are rational, extending the theorem on the rationality of 'manifolds with one apparent double point', proved in [CMR04]. In $\S 3$ we compare various properties of LQELMs and CCMs; see Proposition 3.2. Examples show that the former class is much larger than the first. In particular, using results by Hwang and Kebekus [HK05] (see also [IR07]), we see that complete intersections (of high dimension with respect to the multidegree) are CCM, while the only LQELM which are complete intersections are the hyperquadrics; see Proposition 3.4. We also characterize LQELMs (when $\delta \geqslant 3$ ) in terms of the projective geometry of the variety of lines passing through a general point and contained in $X$; see Proposition 3.5 and [Rus07, Theorem 2.3]. The main properties of LQELMs are collected in Theorem 3.7. In particular, their classification is reduced to the case when the Picard group is generated by the class of the hyperplane section. Conjecturally, QELMs with $\operatorname{Pic}(X) \cong \mathbb{Z}\left\langle\mathcal{O}_{X}(1)\right\rangle$ are linear sections of rational homogeneous manifolds (which are completely classified); see Remark 3.8. The last section contains two applications. The first one, due to $\mathrm{Fu}$ [Fu08], gives a substantial improvement of one of the main results from [KS02] and is based on ideas developed in [Rus07, IR07] and the present paper. The other application is a new proof of the classification, due to Ein [Ein86], of manifolds with small dual. To the best of our knowledge, this is the first time that the classification of dual defective manifolds has been connected to (and, conjecturally, even reduced to, cf. Remark 4.5) that of secant defective mainfolds, via the key concept of (L)QELM.

\section{Preliminaries}

We work over the field of complex numbers. Notation and terminology are the same as in [Rus07]; we recall below some of the relevant facts.

Let $X \subset \mathbb{P}^{N}$ be an irreducible non-degenerate projective variety of dimension $n$. Let

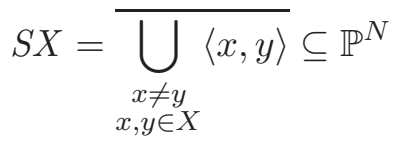

be the secant variety to $X$; see also the construction in (3).

Clearly $\operatorname{dim}(S X) \leqslant \min \{N, 2 n+1\}$. If $\operatorname{dim}(S X)<2 n+1$, then $X \subset \mathbb{P}^{N}$ is said to be secant defective. The secant defect of $X \subset \mathbb{P}^{N}$ is equal to $\delta(X):=2 n+1-\operatorname{dim}(S X)$.

For $p \in S X \backslash X$, the closure of the locus of couples of distinct points of $X$ spanning secant lines passing through $p$ is called the entry locus of $X$ with respect to $p \in S X$ and it will be denoted by $\Sigma_{p}(X)$. The closure of the locus of secant lines to $X$ passing through $p$ is a cone over $\Sigma_{p}(X)$; let us call it $C_{p}(X)$. If $X \subset \mathbb{P}^{N}$ is smooth, then $\Sigma_{p}(X)=C_{p}(X) \cap X$ as schemes for general $p \in S X$; see, for example, [FR81, Lemma 4.5]. Moreover, it is easy to see that for general $p \in S X, \Sigma_{p}(X)$ is equidimensional of dimension equal to $\delta(X)$. Thus $\operatorname{dim}\left(C_{p}(X)\right)=\delta(X)+1$. In general, $\Sigma_{p}(X)$ may be reducible.

Let $X \subset \mathbb{P}^{N}$ be an irreducible non-degenerate projective manifold of dimension $n$ and secant defect $\delta \geqslant 0$.

In the following definition, we consider varieties having the simplest entry locus.

Definition 1.1 (cf. also [KS02, Rus07, IR07]). Let $X$ be as in (1).

(i) $X$ is said to be a quadratic entry locus manifold of type $\delta \geqslant 0$, briefly a QELM of type $\delta$, if for general $p \in S X$ the entry locus $\Sigma_{p}(X)$ is a quadric hypersurface of dimension $\delta$. 


\section{VARIETIES WITH QUADRATIC ENTRY LOCUS, II}

(ii) $X$ is said to be a local quadratic entry locus manifold of type $\delta \geqslant 0$, briefly a LQELM of type $\delta$, if through two general points there passes a quadric hypersurface of dimension $\delta$ contained in $X$.

(iii) $X$ is said to be a conic-connected manifold, briefly a $C C M$, if through two general points of $X$ there passes an irreducible conic contained in $X$.

Note that, for $\delta=0$, being a LQELM imposes no restriction on $X$. Clearly, any QELM is LQEL and any defective LQELM is CC.

Lemma 1.2 (cf. Lemma 1.2 in [Rus07]). Let $X$ be a LQELM with $\delta>0$ and let $x, y \in X$ be general points. There is a unique quadric hypersurface of dimension $\delta$, say $Q_{x, y}$, passing through $x, y$ and contained in $X$. Moreover, $Q_{x, y}$ is irreducible.

The following proposition is easy to prove.

Proposition 1.3. Let $X \subset \mathbb{P}^{N}$ be an irreducible non-degenerate smooth projective variety.

(i) If $X^{\prime} \subset \mathbb{P}^{M}, M \leqslant N-1$, is an isomorphic projection of $X$, then $X^{\prime}$ is a LQELM if and only if $X$ is a $L Q E L M$.

(ii) If $X$ is a (L)QELM of type $\delta \geqslant 1$, then a general hyperplane section is a (L)QELM of type $\delta-1$.

(iii) If $X$ is a $Q E L M$ and $S X=\mathbb{P}^{N}$, then $X$ is linearly normal.

Proof. Parts (i) and (ii) are standard and left to the reader, so that we prove only part (iii). Suppose that $X \subset \mathbb{P}^{N}$ were the isomorphic projection of $\bar{X} \subset \mathbb{P}^{N+1}$ from a point $q \in \mathbb{P}^{N+1} \backslash S \bar{X}$. Since $N=\operatorname{dim}(S X)=\operatorname{dim}(S \bar{X}), S \bar{X}$ would be a hypersurface of degree at least two. A general $p \in S X$ would be the projection from $q$ of at least two different points $p_{1}, p_{2}$ belonging to $\langle q, p\rangle \cap S \bar{X}$. Then the projections of the entry loci $\Sigma_{p_{1}}(\bar{X})$ and $\Sigma_{p_{2}}(\bar{X})$ yield two irreducible components of $\Sigma_{p}(X)$. This is a contradiction, since $\Sigma_{p}(X)$ is a smooth, hence irreducible, $\delta$-dimensional quadric hypersurface; see [FR81, pp. 964-966].

If $x \in X \subset \mathbb{P}^{N}$ is a smooth point, we denote by $\mathbf{T}_{x} X$ the affine Zariski tangent space at $x$ and by $T_{x} X$ its projective closure in $\mathbb{P}^{N}$.

The dimension of the image of the projection of $X \subset \mathbb{P}^{N}$ from a general tangent space to $X$, called the tangential projection of $X \subset \mathbb{P}^{N}$, is easily computed via the Terracini lemma (see, for example, [Rus07, $\S 1]$ ). Let $x \in X \subset \mathbb{P}^{N}$ be a general point and let

$$
\pi_{x}: X \rightarrow W_{x} \subseteq \mathbb{P}^{N-n-1}
$$

be the projection of $X$ from $T_{x} X$. We have $\operatorname{dim}\left(W_{x}\right)=n-\delta$, so that a general fiber of $\pi_{x}$ is of pure dimension $\delta$.

Lemma 1.4 (cf. Lemma 1.6 in [Rus07]). Let $X \subset \mathbb{P}^{N}$ be a smooth irreducible non-degenerate variety, and assume that $\delta>0$. The irreducible components of the closure of a general fibre of $\pi_{x}$ are not linear.

\section{Tangential projections and the geometry of LQELMs}

The main result of this section is the following theorem, generalizing [CMR04, Corollary 4.2], where the case of QELMs of type $\delta=0$ in $\mathbb{P}^{2 n+1}$, i.e. of varieties with one apparent double point, was considered.

Theorem 2.1. Let $X \subset \mathbb{P}^{N}$ be as in (1) and let $x \in X$ be a general point. Then the following hold. 


\section{P. IONESCU AND F. Russo}

(i) If $X$ is a QELM of type $\delta \geqslant 0$, the projection from a general $\delta$-codimensional subspace of $T_{x} X$ passing through $x$ is birational onto its image. In particular, if $S X=\mathbb{P}^{N}$, then $X$ is rational.

(ii) Conversely, if the projection from a general $\delta$-codimensional subspace of $T_{x} X$ passing through $x$ is birational, then $X$ is a LQELM of type $\delta$.

(iii) If $X \subset \mathbb{P}^{N}$ is a LQELM of type $\delta>0$, then $X$ is rational.

We recall some results from [CMR04] (see also [CR06]) in order to point out a relation between the entry loci of a variety and a general fiber of the tangential projection. We believe this relation, contained in Proposition 2.2 below, is interesting in itself.

Let $X \subset \mathbb{P}^{N}$ be an irreducible non-degenerate projective variety and let

$$
S_{X}:=\overline{\{(x, y, z) \mid x \neq y, z \in\langle x, y\rangle\}} \subset X \times X \times \mathbb{P}^{N}
$$

be the abstract secant variety of $X \subset \mathbb{P}^{N}$, which is an irreducible projective variety of dimension $2 n+1$. Let us consider the projections of $S_{X}$ onto the factors $X \times X$ and $\mathbb{P}^{N}$.

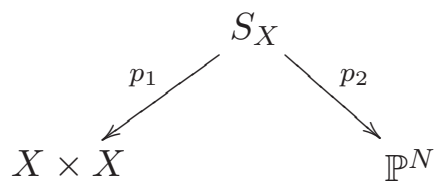

With this notation we get

$$
p_{2}\left(S_{X}\right)=\overline{\bigcup_{\substack{x \neq y \\ x, y \in X}}\langle x, y\rangle}=S X \subseteq \mathbb{P}^{N} .
$$

Let $L=\langle x, y\rangle$ with $x \in X$ and $y \in X$ general points, i.e. $L$ is a general secant line to $X$, and let $p \in\langle x, y\rangle \subseteq S X \subseteq \mathbb{P}^{N}$ be a general point. We fix coordinates on $L$ so that the coordinate of $x$ is 0 ; let $U$ be an open subset of $\mathbb{A}_{\mathbb{C}}^{1} \subset L$ containing $0=x$. Let $p_{2}: S_{X} \rightarrow S X \subseteq \mathbb{P}^{N}$ be as above and let

$$
Z_{U}=p_{2}^{-1}(U) \subset S_{X}
$$

By changing $U$, if necessary, we can suppose that $p_{2}: Z_{U} \rightarrow U$ is flat over $U \backslash\{0\}$ and that $\operatorname{dim}\left(Z_{U}\right)_{t}=\delta(X)$ for every $t \neq 0$. The projection of $p_{1}\left(\left(Z_{U}\right)_{t}\right)$ onto one of the factors is $\Sigma_{t}$, the entry locus of $X$ with respect to $t$ for every $t \neq 0$.

Moreover, by definition, a point $(r, s) \in X \times X, r \neq s$, belongs to $\left(Z_{U}\right)_{t}, t \neq 0$, if and only if $t \in\langle r, s\rangle$, that is if and only if $(r, s) \in p_{2}^{-1}(t)$. Thus, if $\psi_{t}: X \rightarrow \mathbb{P}^{N-1}$ is the projection from $t$ onto a disjoint $\mathbb{P}^{N-1}$, we can also suppose that $\psi_{t}$ is a morphism for every $t \neq 0$ and a rational map not defined at $x=0$ for $t=0$. The above analysis says that the abstract entry locus $\left(Z_{U}\right)_{t}, t \neq 0$, can be considered as the closure in $X \times X$ of the double point locus scheme of $\psi_{t}$, minus the diagonal $\Delta_{X} \subset X \times X$.

Let $T=\left\langle T_{x} X, y\right\rangle$, so that $T$ is a general $\mathbb{P}^{n+1}$ containing $T_{x} X$ and a general point $y \in X$. By definition $\pi_{x}^{-1}\left(\pi_{x}(y)\right)=T \cap X \backslash\left(T_{x} X \cap X\right)$, with the notation as in (2). Let

$$
F_{y}=\overline{\pi_{x}^{-1}\left(\pi_{x}(y)\right)}
$$

be the closure of the fiber of $\pi_{x}$ through $y$. Every irreducible component of $F_{y}$ has dimension $\delta(X)$ by the Terracini lemma and by the generality of $y$; see the discussion after (2). Generic smoothness ensures also that there exists only one irreducible component of $F_{y}$ through $y$.

By using the same ideas as in [CMR04], we have the following result, not explicitly stated in loc. cit., because a slightly different degeneration was considered. For more details about the construction recalled above and below, we refer to [CMR04, $\S \S 3$ and 4] and [CR06, $\S 2]$. 


\section{VARIETIES WITH QUADRATIC ENTRY LOCUS, II}

Proposition 2.2. Let the notation be as above. The closure of the fiber of $\pi_{x}$ through a general point $y \in X$ is contained in the flat limit of the family $\left\{\left(Z_{U}\right)_{t}\right\}_{t \neq 0}$. In other words, the closure of a general fiber of the tangential projection is a degeneration of the general entry locus of $X$.

Proof. We shall look at $\psi_{t}$ as a family of morphisms and study the limit of the double point scheme $\left(Z_{U}\right)_{t}$.

Consider the products $\mathcal{X}=X \times U$ and $\mathbb{P}_{U}=\mathbb{P}^{N-1} \times U$. The projections $\psi_{t}$, for $t \in U$, fit together to give a rational map $\psi: \mathcal{X} \rightarrow \mathbb{P}_{U}$, which is defined everywhere except at the pair $(x, 0)$. In order to extend the projection not defined at $x \in X$, we have to blow up $\mathcal{X}$ at $(x, 0)$. Let $\sigma: \widetilde{\mathcal{X}} \rightarrow \mathcal{X}$ be this blowing-up and let $Z \simeq \mathbb{P}^{n}$ be the exceptional divisor. Looking at the obvious morphism $\varphi: \widetilde{\mathcal{X}} \rightarrow U$, we see that this is a flat family of varieties over $U$. The fiber $\widetilde{\mathcal{X}}_{t}$ over a point $t \in U \backslash\{0\}$ is isomorphic to $X$, whereas the fiber $\widetilde{\mathcal{X}}_{0}$ over $t=0$ is of the form $\widetilde{\mathcal{X}}_{0}=\widetilde{X} \cup Z$, where $\widetilde{X} \rightarrow X$ is the blowing-up of $X$ at $x$, and $\widetilde{X} \cap Z=E$ is the exceptional divisor of this blowing-up, the intersection being transverse. Reasoning as in [CMR04, Lemma 3.1], it is easy to see that $\psi_{0}$ acts on $\widetilde{X}$ as the projection from the point $0=x$, while it maps $Z$ isomorphically onto the linear space $\psi_{0}(T)=\mathbb{P}^{n}$. This immediately implies that every point of $T \cap X$, different from $x$, appears in the 'double point scheme' of $\psi_{0}: \widetilde{X} \cup Z \rightarrow \mathbb{P}^{N-1}$. Therefore $F_{y}$, being of dimension $\delta(X)$, is contained in the flat limit of $\left\{\left(Z_{U}\right)_{t}\right\}_{t \neq 0}$, proving the assertion.

For an irreducible variety $X \subset \mathbb{P}^{N}$ we denote by $\mu(X)$ the number of secant lines passing through a general point of $S X$. If $\delta(X)>0$, then $\mu(X)$ is infinite, while for $\delta(X)=0$ the above number is finite and in this case

$$
\nu(X)=\mu(X) \cdot \operatorname{deg}(S X)
$$

is called the number of apparent double points of $X \subset \mathbb{P}^{N}$. With these definitions we obtain the following generalization of [CMR04, Theorem 4.1] (see also [CR06, Theorem 2.7]).

Theorem 2.3. Let $X \subset \mathbb{P}^{N}$ be as in (1). If $\delta(X)=0$, then

$$
0<\operatorname{deg}\left(\pi_{x}\right) \leqslant \mu(X) .
$$

In particular, for a QELM of type $\delta=0$, the general tangential projection is birational.

If $X \subset \mathbb{P}^{N}$ is a $Q E L M$ of type $\delta>0$, then the general fiber of $\pi_{x}$ is irreducible. More precisely the closure of the fiber of $\pi_{x}$ passing through a general point $y \in X$ is the entry locus of a general point $p \in\langle x, y\rangle$, i.e. a smooth quadric hypersurface.

Proof. If $\delta(X)=0$, then for $t \in U \backslash\{0\}$ the zero-dimensional scheme $\left(Z_{U}\right)_{t}$ has length equal to $2 \mu(X)$. The zero-dimensional scheme $F_{y}$ contains $\operatorname{deg}\left(\pi_{x}\right)$ isolated points, yielding $2 \operatorname{deg}\left(\pi_{x}\right)$ points in the flat limit of $\left\{\left(Z_{U}\right)_{t}\right\}_{t \neq 0}$ by Proposition 2.2 and proving the first part.

Suppose $X$ is a QELM of type $\delta>0$. Then for every $t \neq 0$ the $\delta$-dimensional scheme $\left(Z_{U}\right)_{t}$ is a smooth quadric hypersurface by definition of QELMs. The fiber $F_{y}$ contains the entry locus $\Sigma_{p}$ of a general point $p \in\langle x, y\rangle$, which is a smooth quadric hypersurface of dimension $\delta$ passing through $x$ and $y$. By Proposition 2.2 the variety $F_{y}$ is also contained in the flat limit of $\left\{\left(Z_{U}\right)_{t}\right\}_{t \neq 0}$. Therefore, $F_{y}$ coincides with $\Sigma_{p}$. In fact, in this case the family $\left\{\left(Z_{U}\right)_{t}\right\}_{t \neq 0}$ is constant.

Proof of Theorem 2.1. Suppose that $X \subset \mathbb{P}^{N}$ is a QELM of type $\delta \geqslant 0$. If $\delta=0$ then the first part of Theorem 2.3 yields that $\pi_{x}$ is birational onto its image (see also [CMR04, Corollary 4.2]).

Suppose from now on that $\delta>0$. The projection from a general codimension $\delta$ linear subspace $L \subseteq T_{x} X$ passing through $x$ is a rational map $\pi_{L}: X \rightarrow \mathbb{P}^{N-n+\delta-1}$. For general $y \in X$, the linear space $\langle L, y\rangle$ is obtained by cutting $\left\langle T_{x} X, y\right\rangle$ with $\delta$ general hyperplanes $H_{1}^{y}, \ldots, H_{\delta}^{y}$ passing through $x$ and $y$. From the second part of Theorem 2.3, it follows that, for general $y \in X, \overline{\pi_{x}^{-1}\left(\pi_{x}(y)\right)}$ is a 


\section{P. IOnEsCu AND F. Russo}

$\delta$-dimensional quadric $Q_{x, y}$. Then

$$
\pi_{L}^{-1}\left(\pi_{L}(y)\right) \subseteq Q_{x, y} \cap H_{1}^{y} \cap \cdots \cap H_{\delta}^{y}=\{x, y\},
$$

yielding the birationality of $\pi_{L}$ onto its image. If $S X=\mathbb{P}^{N}$, then $N-n+\delta-1=n$. Part (i) is now completely proved.

Suppose we are in the hypothesis of part (ii). Let $L=\mathbb{P}^{n-\delta} \subset T_{x} X$ be a general linear subspace passing through $x$. Then $L$ is the tangent space of a general codimension $\delta$ linear section of $X \subset \mathbb{P}^{N}$ passing through $x$, let us say $Z$. Thus the restriction of $\pi_{L}$ to $Z, \pi_{L \mid Z}: Z \rightarrow$ $\mathbb{P}^{N-\delta-\operatorname{dim}(Z)-1}=\mathbb{P}^{N-n-1}$, is the projection from $L=T_{x} Z$. Since $\pi_{L}$ restricted to $X$ is birational onto its image, then $\pi_{L \mid Z}$ is easily seen to also be birational onto its image. Moreover, looking at $\pi_{L \mid Z}$ as the projection from $T_{x} Z$, we get $\pi_{L \mid Z}(Z)=\pi_{x}(X)=W_{x} \subseteq \mathbb{P}^{N-n-1}$. Each irreducible component of a general fiber of $\pi_{x}$ produces at least one point in a general fiber of $\pi_{L \mid Z}$. Hence $\pi_{x}: X \rightarrow W_{x} \subseteq \mathbb{P}^{N-n-1}$ has irreducible general fibers of dimension $\delta$ by the birationality of $\pi_{L \mid Z}$

We shall prove inductively that $F_{y}=\overline{\pi_{x}^{-1}\left(\pi_{x}(y)\right)}$ is a $\delta$-dimensional quadric for general $y \in X$. So there is no loss of generality in supposing that $\delta=1$, by passing to a general linear section; see Proposition 1.3. We claim that set theoretically $L \cap F_{y}=\{x\}$. We have $F_{y} \subset\left\langle T_{x} X, y\right\rangle$ so that $T_{x} X \cap F_{y}$ consists of a finite number of points. By the generality of $L$ we get $L \cap F_{y} \subseteq\{x\}$. Let $t=\pi_{x}(y)$. Since $\langle L, t\rangle$ is a hyperplane in $\left\langle T_{x} X, y\right\rangle=\left\langle T_{x} X, t\right\rangle$, intersecting $\pi_{x}^{-1}\left(\pi_{x}(y)\right)$ transversally at a unique point, we get that either we are in the case of the claim or $F_{y}$ is a line. This last case is excluded by Lemma 1.4 .

Let $q=\pi_{L \mid Z}^{-1}(t)=\langle L, t\rangle \cap Z \backslash(L \cap Z)$. By definition,

$$
\langle L, t\rangle=\langle L, q\rangle .
$$

Consider the projection from $t$ onto $T_{x} X$, let us say $\psi_{t}:\left\langle T_{x} X, t\right\rangle-\rightarrow T_{x} X$. Let $\widetilde{F}_{y}=\psi_{t}\left(F_{y}\right)$. By definition $x \in \widetilde{F}_{y}$ because $x \in F_{y}$. Moreover, we claim that $L \cap \widetilde{F}_{y}$ is supported at $x$, so that $\widetilde{F}_{y}$ is a line through $x$. Indeed, if $z \in L \cap \widetilde{F}_{y}$, then there exists $w \in\langle z, t\rangle \cap F_{y} \subset\langle L, t\rangle \cap F_{y}=\langle L, q\rangle \cap F_{y}$, where the last equality follows from (4). Thus either $w=x$ or $w=q$ and in any case $x=\psi_{t}(w)=z$. Therefore, $F_{y} \subset\left\langle\widetilde{F}_{y}, t\right\rangle \simeq \mathbb{P}^{2}$; moreover, the Trisecant lemma implies that the line $\langle x, y\rangle$ cuts transversally $X$, hence also a fortiori $F_{y}$, at $x$ and at $y$. The line $\langle x, y\rangle$ is contained in the plane $\left\langle\widetilde{F}_{y}, t\right\rangle$, so that $\operatorname{deg}\left(F_{y}\right)=2$ and $F_{y}$ is a smooth conic passing through $x$ and $y$, concluding the proof.

Let us prove part (iii). Fix a general point $x \in X$ and denote by $\mathcal{Q}_{x}$ (an irreducible component of) the family of $\delta$-dimensional quadric hypersurfaces contained in $X$ and passing through $x$. Let $\pi: \mathcal{F}_{x} \rightarrow \mathcal{Q}_{x}$ be the universal family and let $\varphi: \mathcal{F}_{x} \rightarrow X$ be the tautological morphism.

Assume first that $\delta=1$. Then $\varphi$ is surjective by definition of a LQELM and birational by Lemma 1.2. Note that $\pi$ has a section, corresponding to the point $x$. Denote by $\mathcal{E} \subset \mathcal{F}_{x}$ the image of this section. Consider the blowing-up $\sigma: X^{\prime} \rightarrow X$ of $X$ at $x$. Since both $\mathcal{Q}_{x}$ and $\pi$ are generically smooth, the birational map $\psi=\sigma^{-1} \circ \varphi$ is defined at a general point of $\mathcal{E}$. Moreover, as $\mathcal{E}$ is contracted by $\varphi$ to the point $x$, then $\psi$ sends $\mathcal{E}$ to $E$, the exceptional divisor of $\sigma$. So we have the following diagram.

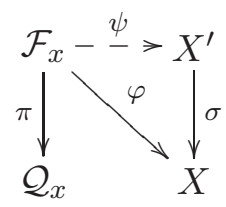




\section{VARIETIES WITH QUADRATIC ENTRY LOCUS, II}

By the Zariski main theorem the map $\psi^{-1}$ is defined at a general point of $E$, and hence $\psi$ induces a birational map $\psi_{0}: \mathcal{E} \rightarrow E=\mathbb{P}^{n-1}$. Thus $\mathcal{F}_{x}$ is birational to $\mathbb{P}^{n}$, being birational to a family of conics with a section over a rational base, and $X$ is a rational variety, as claimed. A general version of the above argument appears in [IN03, Proposition 3.1].

Suppose now that $\delta \geqslant 2$ and fix $H$ a general hyperplane section of $X$ through $x$. Using Lemma 1.2, we see that sending a quadric hypersurface through $x$ to its trace on $H$ yields a birational map between the families $\mathcal{Q}_{x}(X)$ and $\mathcal{Q}_{x}(H)$. So, we see inductively that $\mathcal{Q}_{x}(X)$ is a rational variety of dimension $n-\delta$. Therefore, $\mathcal{F}_{x}$ is rational, as the family $\mathcal{F}_{x} \rightarrow \mathcal{Q}_{x}$ has a section. Being birational to $\mathcal{F}_{x}$ by Lemma $1.2, X$ is rational too.

Remark 2.4. Bronowski claims in [Bro32] that $X \subset \mathbb{P}^{2 n+1}$ is a variety with one apparent double point if and only if the projection of $X$ to $\mathbb{P}^{n}$ from a general tangent space to $X$ is a birational map; he also formulates a generalization to arbitrary secant $k$-planes; see [CR06] for more details. Unfortunately, Bronowski's argument is unclear and we do not know of any convincing proof for this statement. Ciliberto et al. [CMR04, Corollary 4.2] (see also Theorem 2.1) prove one implication showing the rationality of varieties with one apparent double point. The open implication would be a very useful tool for constructing examples.

We can generalize the Bronowski conjecture to the following: a smooth irreducible $n$-dimensional variety $X \subset \mathbb{P}^{2 n+1-\delta(X)}$ is a QELM if and only if the projection from a general codimension $\delta(X)$ linear subspace of $T_{x} X$ passing through $x$ is birational. Theorem 2.1 proves one implication, yielding the rationality of QELMs and extending [CMR04, Corollary 4.2]. One may consult [CR06] for other generalizations of the above conjecture to higher secant varieties.

It is worth mentioning that the above results reveal the following interesting picture for the tangential projections of QELMs of type $\delta \geqslant 0$ with $S X=\mathbb{P}^{N}$ : for $\delta=0$ we project from the whole space and we have varieties with one apparent double point; at the other extreme we found the stereographic projection of quadric hypersurfaces, the only QELMs of type equal to their dimension.

\section{LQEL versus CC}

We recall the following definition from [BBI00].

Definition 3.1 [BBI00]. A smooth rational curve $C \subset X$, where $X$ is a projective manifold of dimension $n$, is a quasi-line if $N_{C \mid X} \simeq \bigoplus_{1}^{n-1} \mathcal{O}_{\mathbb{P}^{1}}(1)$.

The relation between the notions LQELM and CCM is clarified in the following.

Proposition 3.2. Let $X \subset \mathbb{P}^{N}$ be a $C C M$ of secant defect $\delta$. Let $C=C_{x, y}$ be a general conic through the general points $x, y \in X$ and let $c$ be the point representing $C$ in the Hilbert scheme of $X$. Let $\mathcal{C}_{x}$ be the unique irreducible component of the Hilbert scheme of conics passing through $x$ which contains the point $c$.

(i) We have $n+\delta \geqslant-K_{X} \cdot C=\operatorname{dim}\left(\mathcal{C}_{x}\right)+2 \geqslant n+1$.

(ii) The equality $-K_{X} \cdot C=n+\delta$ holds if and only if $X \subset \mathbb{P}^{N}$ is a LQELM.

(iii) The following conditions are equivalent:

(a) $\operatorname{dim}\left(\mathcal{C}_{x}\right)=n-1$;

(b) $C$ is a quasi-line;

(c) all conics through $x, y$ are non-degenerate.

(iv) If $\delta \geqslant 3$, then $X \subset \mathbb{P}^{N}$ is a Fano manifold with $\operatorname{Pic}(X) \simeq \mathbb{Z}\left\langle\mathcal{O}_{X}(1)\right\rangle$ and index $i(X)=$ $\left(\operatorname{dim}\left(\mathcal{C}_{x}\right) / 2\right)+1$. 


\section{P. IONESCU AND F. Russo}

Proof. We have the universal family $g: \mathcal{F}_{x} \rightarrow \mathcal{C}_{x}$ and the tautological morphism $f: \mathcal{F}_{x} \rightarrow X$, which is surjective. Since $C \in \mathcal{C}_{x}$ is a general conic and since $x \in X$ is general, we get $\operatorname{dim}\left(\mathcal{C}_{x}\right)=-K_{X} \cdot C-2$.

Take a general point $y \in X$ and a general $p \in\langle x, y\rangle$. The conics passing through $x$ and $y$ are parameterized by $g\left(f^{-1}(y)\right)$, which has pure dimension

$$
\operatorname{dim}\left(\mathcal{F}_{x}\right)-n=\operatorname{dim}\left(\mathcal{C}_{x}\right)+1-n=-K_{X} \cdot C-1-n .
$$

We claim that the locus of conics through $x$ and $y$, denoted by $\mathcal{L}_{x, y}$, has dimension $-K_{X} \cdot C-n$ and is clearly contained in the irreducible component of the entry locus (with respect to $p$ ) through $x$ and $y$. Indeed, conics through $x, y$ and another general point $z \in \mathcal{L}_{x, y}$ have to be finitely many. Otherwise, their locus would fill up the plane $\langle x, y, z\rangle$ and this would imply that the line $\langle x, y\rangle$ is contained in $X$. However, we have excluded linear spaces from the definition of CCMs and LQELMs. Therefore, $\delta \geqslant-K_{X} \cdot C-n$, that is, $-K_{X} \cdot C \leqslant n+\delta$.

The locus of conics through $x$ and $y$ is contained in $\left\langle T_{x} X, y\right\rangle \cap\left\langle x, T_{y} X\right\rangle$, which is a linear space of dimension $\delta+1$. Indeed, by the Terracini lemma,

$$
\operatorname{dim}\left(T_{x} X \cap T_{y} X\right)=2 n-\operatorname{dim}(S X)=\delta-1 .
$$

By the Trisecant lemma, $x \notin T_{y} X$ and $y \notin T_{x} X$, so that

$$
\operatorname{dim}\left(\left\langle T_{x} X, y\right\rangle \cap\left\langle x, T_{y} X\right\rangle\right)=\delta+1 .
$$

If $-K_{X} \cdot C=n+\delta$, then, for $p \in\langle x, y\rangle$ general, the irreducible component $\Sigma_{x, y}^{p}$ of the entry locus passing through $x$ and $y$ coincides with the locus of conics through $x$ and $y$. Thus $\Sigma_{x, y}^{p}$ is a quadric hypersurface by the Trisecant lemma and by the generality of $x$ and $y$ (if $\delta=n, X \subset \mathbb{P}^{n+1}$ is a quadric hypersurface). So, part (ii) is proved.

Next we see part (iii). Conditions (a) and (b) are equivalent, since the normal bundle of $C$ in $X$ is ample, of degree $\operatorname{dim}\left(\mathcal{C}_{x}\right)$.

Assume that condition (a) holds. The dimension of the subfamily consisting of reducible conics from $\mathcal{C}_{x}$ has dimension at most $n-2$. Hence their locus is of dimension at most $n-1$ and does not contain the general point $y$. So we have condition (c).

Assume that condition (c) holds. Then, by bend and break there are finitely many conics through $x$ and $y$, giving condition (a).

Finally, part (iv) follows from the Barth-Larsen theorem, the fact that $X$ contains moving conics and part (i).

Examples 3.3. (i) For $n \geqslant 3$, let $X$ be a smooth cubic hypersurface in $\mathbb{P}^{n+1}$ or the smooth complete intersection of two hyperquadrics in $\mathbb{P}^{n+2}$. Use, for example, [BBI00, Theorem 3.2] and induction on $n$ to see that $X$ is CC. In the first case, $X$ is a hypersurface of degree three so it cannot be a LQELM. In the second case, $\delta(X)=n-1$; as the Picard group is generated by the hyperplane section, $X$ cannot contain hyperquadrics of dimension $n-1$.

(ii) CCMs $X \subset \mathbb{P}^{N+1}$ of secant defect $\delta(X)=\delta-1 \geqslant 2$, constructed from QELMs $Z \subset \mathbb{P}^{N}$ of type $\delta \geqslant 3$.

Let $Z \subset \mathbb{P}^{N}$ be a QELM of type $\delta \geqslant 3$. Consider $\mathbb{P}^{N}$ as a hyperplane in $\mathbb{P}^{N+1}$, take $q \in \mathbb{P}^{N+1} \backslash \mathbb{P}^{N}$ and let $W=C_{q}(Z) \subset \mathbb{P}^{N+1}$ be the cone over $Z$ of vertex $q$. Let $X=C_{q}(Z) \cap Q \subset \mathbb{P}^{N+1}$, where $Q \subset \mathbb{P}^{N+1}$ is a general quadric hypersurface. Then $S X=C_{q}(S Z)$, yielding $\delta(X)=\delta(Z)-1 \geqslant 2$. Moreover, $\operatorname{Pic}(X) \simeq \mathbb{Z}\left\langle\mathcal{O}_{X}(1)\right\rangle$ and $X \subset \mathbb{P}^{N+1}$ is a CCM of index $i(X)=(n+\delta(X)-1) / 2$.

Indeed, let $\pi: T=\mathbb{P}\left(\mathcal{O}_{Z} \oplus \mathcal{O}_{Z}(1)\right) \rightarrow Z$ and let $E \subset T$ be the section at infinity of $\pi$. If $\varphi: T=\mathbb{P}\left(\mathcal{O}_{Z} \oplus \mathcal{O}_{Z}(1)\right) \rightarrow \mathbb{P}^{N+1}$ is the tautological morphism given by (a sublinear system of) $\left|\mathcal{O}_{T}(1)\right|$, then $\varphi(T)=W$ and $X \subset \mathbb{P}^{N+1}$ can be naturally thought of as an element of $\left|\mathcal{O}_{T}(2)\right|$. Recall that $\varphi$ restricts to an isomorphism between $T \backslash E$ and $W \backslash\{q\}$. By adjunction we get that 
$\omega_{X}=\mathcal{O}_{X}(1-i(Z))$, that is,

$$
i(X)=i(Z)-1=\frac{n+\delta}{2}-1=\frac{n+\delta(X)-1}{2} .
$$

In particular, $X \subset \mathbb{P}^{N+1}$ is a Fano manifold. Adjunction formula also says that the double cover $\pi_{q}: X \rightarrow Z$, induced by the projection from $q, \pi_{q}: \mathbb{P}^{N+1} \backslash\{q\} \rightarrow \mathbb{P}^{N}$, is ramified along a hyperplane section of $Z \subset \mathbb{P}^{N}$.

Take two general points $x, y \in X$ and let $x^{\prime}=\pi_{q}(x)$ and $y^{\prime}=\pi_{q}(y)$. Through $x^{\prime}$ and $y^{\prime}$ there passes a smooth quadric hypersurface $Q^{\prime} \subset \mathbb{P}^{\delta+1}$ of dimension $\delta \geqslant 3$. Let $\widetilde{Q}=C_{q}\left(Q^{\prime}\right) \subset \mathbb{P}^{\delta+2}$ and let $\widetilde{X}=C_{q}\left(Q^{\prime}\right) \cap Q \subset \mathbb{P}^{\delta+2}$. Then $\widetilde{X} \subset \mathbb{P}^{\delta+2}$ is a (smooth) complete intersection of two quadric hypersurfaces passing through $x$ and $y$, so that $X \subset \mathbb{P}^{N+1}$ is a CCM since $\tilde{X} \subset \mathbb{P}^{\delta+2}$ is CC by part (i) above.

(iii) When $\delta=1$, CCMs and LQELMs are the same. In this case a general conic through two general points is a quasi-line. Examples are easy to construct, e.g. $X=G \cap H_{1} \cap H_{2} \cap H_{3}$, where $G$ is the Grassmannian of lines in $\mathbb{P}^{m}, m \geqslant 4$, and $H_{i}$ are general hyperplane sections of its Plücker embedding.

(iv) Assume that $\delta=2$ and $X$ is CC. Let $C$ be a general conic passing through two general points. Then $X$ is a LQELM if and only if $C$ is not a quasi-line; see Proposition 3.2. Examples of the LQEL case are given by taking $X=G \cap H_{1} \cap H_{2}$, where $G$ and $H_{i}$ are as above. Examples of the case where $X$ contains quasi-lines are got by applying the construction in part (ii) above starting with $Z=G \cap H$, a hyperplane section of the same Grassmannian.

Many examples of CCMs which are not LQEL come from the following.

Proposition 3.4 (cf. Corollary 2.5 in [IR07]).

(i) If $X \subset \mathbb{P}^{n+r}$ is a smooth non-degenerate complete intersection of multi-degree $\left(d_{1}, d_{2}, \ldots, d_{r}\right)$ with $n>3\left(\sum_{1}^{r} d_{i}-r-1\right)$, then $X$ is a $C C M$.

(ii) If $X$ is a secant defective LQELM and a complete intersection, then $X$ is a hyperquadric.

Proof. Part (i) is exactly [IR07, Corollary 2.5].

We show part (ii). If $n=2$ use [Rus07, Proposition 3.3] to conclude. If $n \geqslant 3$, by the Lefschetz theorem, we have $\operatorname{Pic}(X) \cong \mathbb{Z}\left\langle\mathcal{O}_{X}(1)\right\rangle$ and Proposition 3.2 gives $i(X)=(n+\delta) / 2$. Let $r=N-n$. We have $2 n+1-\delta=\operatorname{dim}(S X) \leqslant N=n+r$, so $\delta \geqslant n+1-r$. Assuming $X$ to be a complete intersection of type $\left(d_{1}, \ldots, d_{r}\right)$, with $d_{i} \geqslant 2$ for all $i$, we get $i(X)=n+r+1-\sum_{1}^{r} d_{i}$; hence

$$
n+2 r+2-2 \sum_{1}^{r} d_{i}=2 i(X)-n=\delta \geqslant n+1-r \quad \text { and } \quad 4 r \leqslant 2 \sum_{1}^{r} d_{i} \leqslant 3 r+1,
$$

so $r=1$.

The proof of the following criterion for recognizing LQELMs illustrates the role of CCMs. Recall from [Rus07] that, if $x \in X$ is a general point, we denote by $Y_{x} \subset \mathbb{P}\left(\mathbf{T}_{x}^{*} X\right)$ the variety of lines through $x$, contained in $X$.

Proposition 3.5. Let $X \subset \mathbb{P}^{N}$ be as in (1) and assume that $\delta \geqslant 3$. The following assertions are equivalent:

(i) $X$ is a LQELM;

(ii) if $x \in X$ is a general point, then $\operatorname{dim}\left(Y_{x}\right) \geqslant(n+\delta) / 2-2$ and $S Y_{x}=\mathbb{P}^{n-1}$. 


\section{P. IONESCU AND F. Russo}

Proof. The fact that part (i) implies (ii) was shown in [Rus07, Theorem 2.3] and, in fact, equality holds in part (ii).

Assume part (ii). $\delta \geqslant 3$ implies via the Barth-Larsen theorem that $\operatorname{Pic}(X) \cong \mathbb{Z}\left\langle\mathcal{O}_{X}(1)\right\rangle$. Moreover,

$$
i(X)=\operatorname{dim}\left(Y_{x}\right)+2 \geqslant \frac{n+\delta}{2} \geqslant \frac{n+3}{2} .
$$

So we may apply [HK05, Theorem 3.14] to deduce that $X$ is a CCM. In the notation of Proposition 3.2, we have $2 i(X)=-K_{X} \cdot C \geqslant n+\delta$. Therefore, $X$ is a LQELM by combining parts (i) and (ii) of Proposition 3.2.

The following proposition is an application of [Rus07, Theorem 2.3].

Proposition 3.6. Assume that $X$ is a LQELM of type $\delta<n$.

(i) If $\delta>n / 2$ then $\delta \leqslant 6$ and $n \leqslant 10$. These cases are classified in [Rus07, Corollary 3.1].

(ii) If $\delta>n / 3$ then $\delta \leqslant 10$ and $n \leqslant 26$. These cases may also be classified by the same method.

(iii) If $k \geqslant 4, \delta>n / k$, then $\delta \leqslant 2(k+2)$.

Proof. See [Rus07, Corollary 3.1] for part (i).

Assume $n / 3<\delta \leqslant n / 2$. Using [Rus07, Theorem 2.8], we find $\delta \leqslant 10$ and the following possibilities for the pairs $(n, \delta):(2,1),(4,2),(5,2),(7,3),(8,4),(10,4),(13,5),(14,6),(15,7),(16,8),(25,9)$ and $(26,10)$.

To prove part (iii) we proceed by induction on $k \geqslant 3$. We may suppose that $\delta \geqslant 10$. Assume that we have $n / k \geqslant \delta>n /(k+1)$ and $k \geqslant 4$. In the notation of [Rus07, Theorem 2.4], we let $X_{1}=Y_{x}$, $\delta_{1}=\delta\left(X_{1}\right)=\delta-2, n_{1}=\operatorname{dim}\left(X_{1}\right)=(n+\delta) / 2-2$. If $\delta_{1}>n_{1} / k$ we get $\delta \leqslant 2(k+3)$, completing the induction. If $\delta_{1} \leqslant n_{1} / k$ it follows that

$$
\delta \leqslant \frac{n+4(k-1)}{2 k-1}, \quad \text { so } \quad \frac{n}{k+1}<\frac{n+4(k-1)}{2 k-1} .
$$

This gives

$$
10 k \leqslant \delta k \leqslant n<\frac{4\left(k^{2}-1\right)}{k-2}
$$

so $3 k^{2}-10 k+2<0$. Therefore, $k=3$; a contradiction.

Consider the following list of examples of QELMs.

(i) $\nu_{2}\left(\mathbb{P}^{n}\right) \subset \mathbb{P}^{n(n+3) / 2}$.

(ii) The projection of $\nu_{2}\left(\mathbb{P}^{n}\right)$ from the linear space $\left\langle\nu_{2}\left(\mathbb{P}^{s}\right)\right\rangle$, where $\mathbb{P}^{s} \subset \mathbb{P}^{n}$ is a linear subspace; equivalently $X \simeq \operatorname{Bl}_{\mathbb{P} s}\left(\mathbb{P}^{n}\right)$ embedded in $\mathbb{P}^{N}$ by the linear system of quadric hypersurfaces of $\mathbb{P}^{n}$ passing through $\mathbb{P}^{s}$; alternatively $X \simeq \mathbb{P}_{\mathbb{P}^{r}}(\mathcal{E})$ with $\mathcal{E} \simeq \mathcal{O}_{\mathbb{P}^{r}}(1)^{\oplus n-r} \oplus \mathcal{O}_{\mathbb{P}^{r}}(2), r=1,2, \ldots, n-1$, embedded by $\left|\mathcal{O}_{\mathbb{P}(\mathcal{E})}(1)\right|$. Here $N=n(n+3) / 2-\left(\begin{array}{c}s+2 \\ 2\end{array}\right)$ and $s$ is an integer such that $0 \leqslant s \leqslant n-2$.

(iii) A hyperplane section of the Segre embedding $\mathbb{P}^{a} \times \mathbb{P}^{b} \subset \mathbb{P}^{N+1}$. Here $n \geqslant 3$ and $N=a b+a+b-1$, where $a \geqslant 2$ and $b \geqslant 2$ are such that $a+b=n+1$.

(iv) $\mathbb{P}^{a} \times \mathbb{P}^{b} \subset \mathbb{P}^{a b+a+b}$ Segre embedded, where $a, b$ are positive integers such that $a+b=n$.

The essential properties of LQELMs are collected in the next theorem. It follows by putting together Theorem 2.1(iii), [IR07, Theorem 2.2] and [Rus07, Theorem 2.3(4d)].

Theorem 3.7. Let $X \subset \mathbb{P}^{N}$ be a defective LQELM. Then $X$ is Fano and rational. Moreover, either $X$ is an isomorphic projection of one of the manifolds listed immediately above or $\operatorname{Pic}(X) \cong$ $\mathbb{Z}\left\langle\mathcal{O}_{X}(1)\right\rangle$ and $i(X)=(n+\delta) / 2$. If $\delta \geqslant 3$, then $Y_{x} \subset \mathbb{P}^{n-1}$ is a QELM of type $\delta-2$, dimension $(n+\delta) / 2-2$ and such that $S Y_{x}=\mathbb{P}^{n-1}$. 


\section{VARIETIES WITH QUADRATIC ENTRY LOCUS, II}

Remark 3.8. Via the above theorem, the classification of secant defective QELMs is reduced to the case where the Picard group is $\mathbb{Z}$. Let us say that $X \subset \mathbb{P}^{N}$ is maximal if $X$ is not a hyperplane section of some (non-degenerate) manifold $X^{\prime} \subset \mathbb{P}^{N+1}$. The following tempting conjecture would, if true, lead to a complete classification of defective QELMs.

Conjecture. Any maximal defective QELM with Picard group $\mathbb{Z}$ is homogeneous.

Note that homogeneous manifolds arising from irreducible representations of (semi)simple complex algebraic groups are QELMs (see [Zak93, ch. III] and [Kaj99]), and the secant defective ones are classified completely (see loc. cit.). Moreover, the results in [Rus07, §3] confirm the conjecture for $\delta>n / 2$. The next two finiteness results for QELMs with $\delta<n$ would follow from the above conjecture:

(i) $\delta \leqslant 8$;

(ii) if $\delta \geqslant 5$, then $n \leqslant 16$.

Proposition 3.6 may be seen as supporting these finiteness expectations. Also, in [Fu08, Corollary 2], it is proved that $\delta \leqslant(n+8) / 3$ holds for any LQELM with $\delta<n$. In particular, result (i) follows from result (ii).

\section{Two applications}

The first application is due to $\mathrm{Fu}$ [Fu08], who found it by relying on the ideas and techniques from [Rus07], [IR07] and Proposition 3.2. We mention his result in order to illustrate the usefulness of our point of view.

TheOREM 4.1 (cf. Theorem 2 in [Fu08]). Let $X$ be as in (1) and assume that $X$ is swept out by hyperquadrics of dimension greater than $[n / 2]+1$, all passing through a fixed point $x \in X$. Then $X$ is a hyperquadric.

This result substantially improves the main application in [KS02], with a much shorter proof.

Our second application concerns the classification of manifolds with small duals.

For an irreducible variety $Z \subset \mathbb{P}^{N}$, we define $\operatorname{def}(Z)=N-1-\operatorname{dim}\left(Z^{*}\right)$ as the dual defect of $Z \subset \mathbb{P}^{N}$, where $Z^{*} \subset \mathbb{P}^{N *}$ is the dual variety of $Z \subset \mathbb{P}^{N}$. In [Ein86, Theorem 2.4] it is proved that if $\operatorname{def}(X)>0$, then $\operatorname{def}(X) \equiv n(\bmod 2)$, a result usually attributed to Landman; see the Introduction of [Ein86]. Moreover, the Zak theorem on tangencies implies that $\operatorname{dim}\left(X^{*}\right) \geqslant \operatorname{dim}(X)$ for a smooth non-degenerate variety $X \subset \mathbb{P}^{N}$; see [Zak93, I.2.5].

We combine the geometry of CCMs and LQELMs to give a new proof of [Ein86, Theorem 4.5]. Our approach avoids the use of Beilinson spectral sequences and more sophisticated computations as in [Ein86, Lemmas 4.2, 4.3 and Theorem 4.4].

We begin by recalling some basic facts from [Ein86].

Proposition 4.2. Let $X \subset \mathbb{P}^{N}$ be as in (1) and assume that $\operatorname{def}(X)>0$. Then:

(i) [Ein86, Theorem 2.4] through a general point $x \in X$ there passes a line $L_{x} \subset X$ such that $-K_{X} \cdot L_{x}=(n+\operatorname{def}(X)+2) / 2$, so that $\operatorname{def}(X) \equiv n(\bmod 2)$;

(ii) [Ein86, Theorem 3.2] $\operatorname{def}(X)=n-2$ if and only if $X \subset \mathbb{P}^{N}$ is a scroll over a smooth curve, i.e. it is a $\mathbb{P}^{n-1}$-bundle over a smooth curve, whose fibers are linearly embedded.

The following proposition reinterprets the result of Hwang and Kebekus [HK05, Theorem 3.14] on Fano manifolds with large index. 


\section{P. IONESCU AND F. Russo}

Proposition 4.3 (cf. [HK05]; see also Proposition 2.4 in [IR07]). Let $X \subset \mathbb{P}^{N}$ be as in (1). Assume that $X$ is a Fano manifold with $\operatorname{Pic}(X) \cong \mathbb{Z}\left\langle\mathcal{O}_{X}(1)\right\rangle$ and let $x \in X$ be a general point.

(i) If $i(X)>2 n / 3$, then $X$ is a $C C M$ with $\delta>n / 3$.

(ii) If $\operatorname{def}(X)>0$ and $\operatorname{def}(X)>(n-6) / 3$, then $X$ is a $C C M$ with $\delta \geqslant \operatorname{def}(X)+2$. Moreover, if $\delta=\operatorname{def}(X)+2$, then $X$ is a LQELM of type $\delta=\operatorname{def}(X)+2$.

(iii) If $X$ is a LQELM of type $\delta$ and $\operatorname{def}(X)>0$, then $\delta=\operatorname{def}(X)+2$.

Proof. See [IR07, Proposition 2.4] for the proof of part (i).

In the hypothesis of part (ii), [Ein86, Theorem 2.4] yields $i(X)=(n+\operatorname{def}(X)+2) / 2>2 n / 3$ so that $X$ is a CCM by part (i). Proposition 3.2 yields $\delta \geqslant \operatorname{def}(X)+2$ and also the remaining assertions of parts (ii) and (iii).

We recall that according to the Hartshorne conjecture, if $n>\frac{2}{3} N$, then $X \subset \mathbb{P}^{N}$ should be a complete intersection and that complete intersections have no dual defect. Thus, assuming the Hartshorne conjecture, the following result yields the complete list of manifolds $X \subset \mathbb{P}^{N}$ such that $\operatorname{dim}\left(X^{*}\right)=\operatorname{dim}(X)$. The second part says that under the LQEL hypothesis the same results hold without any restriction (see also Remark 4.5 below).

Theorem 4.4. Let $X \subset \mathbb{P}^{N}$ be as in (1) and assume that $\operatorname{dim}(X)=\operatorname{dim}\left(X^{*}\right)$.

(i) [Ein86, Theorem 4.5] If $N \geqslant 3 n / 2$, then $X$ is projectively equivalent to one of the following:

(a) a smooth hypersurface $X \subset \mathbb{P}^{n+1}, n=1,2$;

(b) a Segre variety $\mathbb{P}^{1} \times \mathbb{P}^{n-1} \subset \mathbb{P}^{2 n-1}$;

(c) the Plücker embedding $\mathbb{G}(1,4) \subset \mathbb{P}^{9}$;

(d) the 10-dimensional spinor variety $S^{10} \subset \mathbb{P}^{15}$.

(ii) If $X$ is a LQELM, then it is projectively equivalent either to a smooth quadric hypersurface $Q \subset \mathbb{P}^{n+1}$ or to a variety as in (b), (c), (d) above.

Proof. Clearly $\operatorname{def}(X)=0$ if and only if $X \subset \mathbb{P}^{n+1}$ is a hypersurface, giving respectively case (a) or that of a smooth quadric hypersurface. From now on we suppose that $\operatorname{def}(X)>0$ and hence $n \geqslant 3$. By parts (i) and (ii) of Proposition $4.2, \operatorname{def}(X)=n-2$ and $N=2 n-1$ if and only if we are in case (b); see also [Ein86, Theorem 3.3, part c].

Thus, we may assume that $0<\operatorname{def}(X) \leqslant n-4$, that is, $N \leqslant 2 n-3$. Therefore, $\delta \geqslant 4$ and $X$ is a Fano manifold with $\operatorname{Pic}(X) \cong \mathbb{Z}\left\langle\mathcal{O}_{X}(1)\right\rangle$. Moreover, in case (i), $\operatorname{def}(X)=N-n-1>(n-6) / 3$ by hypothesis. Thus Proposition 4.3 yields that $X$ is also a CCM with $\delta \geqslant \operatorname{def}(X)+2$. Also taking into account the last part of Proposition 4.3, from now on we can suppose that $X$ is a CCM with $\delta \geqslant \operatorname{def}(X)+2 \geqslant 3$

We have $n-\delta \leqslant N-1-n=\operatorname{def}(X) \leqslant \delta-2$, that is, $\delta \geqslant n / 2+1$. The Zak linear normality theorem implies $S X=\mathbb{P}^{N}$, so that

$$
N=\operatorname{dim}(S X)=2 n+1-\delta \leqslant \frac{3 n}{2} .
$$

Since $N \geqslant 3 n / 2$, we get $N=3 n / 2, \delta=n / 2+1=\operatorname{def}(X)+2$ and $n$ even. Therefore, $X$ is a LQELM of type $\delta=n / 2+1$ by Proposition 4.3. Using [Rus07, Corollary 3.1] concludes the proof, yielding cases $(\mathrm{c})$ and $(\mathrm{d})$.

Remark 4.5. Let $X \subset \mathbb{P}^{N}$ be as in (1). Assume that $\operatorname{def}(X)>0$ and $\operatorname{Pic}(X) \cong \mathbb{Z}\left\langle\mathcal{O}_{X}(1)\right\rangle$. We conjecture that $X$ is a LQELM and even a QELM if, moreover, it is assumed to be linearly normal. Combined with Remark 3.8, this would imply that maximal dual defective manifolds with $\operatorname{Pic}(X) \cong$ $\mathbb{Z}\left\langle\mathcal{O}_{X}(1)\right\rangle$ are homogeneous, as already conjectured in [BS95]. 


\section{VARIETIES WITH QUADRATIC ENTRY LOCUS, II}

Our last application is the following.

Proposition 4.6. Let $X \subset \mathbb{P}^{N}$ be as in (1), with $\operatorname{Pic}(X) \cong \mathbb{Z}\left\langle\mathcal{O}_{X}(1)\right\rangle$ and $\operatorname{def}(X)>0$. Assume, moreover, that $\operatorname{def}(X)>(n-6) / 3$ and $N \geqslant(5 n+2) / 3$. Then $S X \neq \mathbb{P}^{N}$.

Proof. By Proposition 4.3, $X$ is a CCM. Assume that $S X=\mathbb{P}^{N}$. We get

$$
N=\operatorname{dim}(S X)=2 n+1-\delta \geqslant \frac{5 n+2}{3},
$$

so $\delta \leqslant(n+1) / 3$. It follows that $3 \operatorname{def}(X) \geqslant n-5 \geqslant 3 \delta-6$. By Proposition $4.3, X$ is a LQELM of type $\delta=(n+1) / 3$ and $\operatorname{def}(X)=\delta-2$. From the list in the proof of Proposition 3.6(ii) it follows that $\delta \leqslant 2$, so $\operatorname{def}(X)=0$. This is a contradiction.

\section{ACKNowledGements}

Both authors are grateful to the organizers of the Conference 'Projective varieties with unexpected properties', that took place in Siena, 8-13 June 2004. Our collaboration started while taking part in this very pleasant and fruitful mathematical event.

\section{REFERENCES}

BBI00 L. Bădescu, M. C. Beltrametti and P. Ionescu, Almost-lines and quasi-lines on projective manifolds, in Complex Analysis and Algebraic Geometry (in memory of Michael Schneider) (de Gruyter, Berlin, 2000), 1-27.

BL72 W. Barth and M. E. Larsen, On the homotopy groups of complex projective algebraic manifolds, Math. Scand. 30 (1972), 88-94.

BS95 M. C. Beltrametti and A. J. Sommese, The adjunction theory of complex projective varieties, de Gruyter Exp. Math., vol. 16 (de Gruyter, Berlin, 1995).

Bro32 J. Bronowski, The sum of powers as canonical expressions, Proc. Cambridge Philos. Soc. 29 (1932), 69-82.

CMR04 C. Ciliberto, M. Mella and F. Russo, Varieties with one apparent double point, J. Algebraic Geom. 13 (2004), 475-512.

CR06 C. Ciliberto and F. Russo, Varieties with minimal secant degree and linear systems of maximal dimension on surfaces, Adv. Math. 200 (2006), 1-50.

Deb01 O. Debarre, Higher-Dimensional Algebraic Geometry, Universitext (Springer, Berlin, 2001).

Ein86 L. Ein, Varieties with small dual variety. I, Invent. Math. 86 (1986), 63-74.

Fu08 B. Fu, Inductive characterizations of hyperquadrics, Math. Ann. 340 (2008), 185-194.

FR81 T. Fujita and J. Roberts, Varieties with small secant varieties: the extremal case, Amer. J. Math. 103 (1981), 953-976.

Hwa01 J. M. Hwang, Geometry of minimal rational curves on Fano manifolds, in School on Vanishing Theorems and Effective Results in Algebraic Geometry, Trieste, 2000, ICTP Lecture Notes, vol. 6 (Abdus Salam Int. Cent. Theoret. Phys., 2001), 335-393.

HK05 J. M. Hwang and S. Kebekus, Geometry of chains of minimal rational curves, J. reine angew. Math. 584 (2005), 173-194.

IN03 P. Ionescu and D. Naie, Rationality properties of manifolds containing quasi-lines, Internat. J. Math. 14 (2003), 1053-1080.

IR07 P. Ionescu and F. Russo, Conic-connected manifolds, Preprint (2006), math. AG/0701885.

Kaj99 H. Kaji, Homogeneous projective varieties with degenerate secants, Trans. Amer. Math. Soc. 351 (1999), 533-545.

Kol96 J. Kollár, Rational Curves on Algebraic Varieties, Ergeb. Math. Grenzgeb. (3), vol. 32 (Springer, Berlin, 1996). 


\section{VARIETIES WITH QUADRATIC ENTRY LOCUS, II}

KMM92 J. Kollár, Y. Miyaoka and S. Mori, Rationally connected varieties, J. Algebraic Geom. 1 (1992), 429-448.

KS02 Y. Kachi and E. Sato, Segre's reflexivity and an inductive characterization of hyperquadrics, Mem. Amer. Math. Soc. 160 (2002).

Mor79 S. Mori, Projective manifolds with ample tangent bundle, Ann. of Math. (2) 110 (1979), 593-606.

Mor82 S. Mori, Threefolds whose canonical bundles are not numerically effective, Ann. of Math. (2) 116 (1982), 133-176.

Rus07 F. Russo, Varieties with quadratic entry locus. I, Preprint (2006), math. AG/0701889.

Sco08 G. Scorza, Sulla determinazione delle varietà a tre dimensioni di $S_{r}(r \geqslant 7)$ i cui $S_{3}$ tangenti si tagliano a due a due, Rend. Circ. Mat. Palermo 25 (1908), 193-204; contained in Opere Scelte, vol. I (Edizioni Cremonese, 1960), 167-182.

Sco09 G. Scorza, Sulle varietà a quattro dimensioni di $S_{r}(r \geqslant 9)$ i cui $S_{4}$ tangenti si tagliano a due a due, Rend. Circ. Mat. Palermo 27 (1909), 148-178; contained in Opere Scelte, vol. I (Ed. Cremonese, 1960), 252-292.

Sev01 F. Severi, Intorno ai punti doppi impropri di una superficie generale dello spazio a quattro dimensioni e ai suoi punti tripli apparenti, Rend. Circ. Mat. Palermo 15 (1901), 33-51.

Zak93 F. L. Zak, Tangents and secants of algebraic varieties, Translations in Mathematical Monographs, vol. 127 (American Mathematical Society, Providence, RI, 1993).

Paltin Ionescu ionescu@dima.unige.it

University of Bucharest and Institute of Mathematics of the Romanian Academy

Current address: Dipartimento di Matematica, Università degli Studi di Genova, Via Dodecaneso, 35, 16146 Genova, Italy

Francesco Russo frusso@dmi.unict.it

Dipartimento di Matematica e Informatica, Università degli Studi di Catania, Viale A. Doria 6, 95125 Catania, Italy 